\title{
In-hospital measurement of left ventricular ejection fraction and one-year outcomes in acute coronary syndromes: results from the IMMEDIATE Trial
}

Jayanta T. Mukherjee ${ }^{1,4,8}$, Joni R. Beshansky ${ }^{3,7}$, Robin Ruthazer ${ }^{3,4}$, Hadeel Alkofide ${ }^{1,6}$, Madhab Ray ${ }^{1}$, David Kent ${ }^{3}$, Warren J. Manning ${ }^{4}$, Gordon S. Huggins ${ }^{2}$ and Harry P. Selker; ${ }^{3,5^{*}}$

\begin{abstract}
Background: In patients with acute coronary syndrome (ACS), reduced left ventricular ejection fraction (LVEF) is a known marker for increased mortality. However, the relationship between LVEF measured during index ACS hospitalization and mortality and heart failure (HF) within 1 year are less well-defined.

Methods: We performed a retrospective analysis of 445 participants in the IMMEDIATE Trial who had LVEF measured by left ventriculography or echocardiogram during hospitalization.

Results: Adjusting for age and coronary artery disease (CAD) history, lower LVEF was significantly associated with 1-year mortality or hospitalization for HF. For every $5 \%$ LVEF reduction, the hazard ratio [HR] was 1.26 (95 \% Cl 1.15, 1.38, $P<0.001$ ). Participants with LVEF $<40 \%$ had higher hazard of 1-year mortality or HF hospitalization than those with LVEF > 40 (HR 3.59; $95 \% \mathrm{Cl} 2.05,6.27, P<0.001)$. The HRs for the association of LVEF with the study outcomes were similar whether measured by left ventriculography or by echocardiography, (respectively, HR 1.32; $95 \% \mathrm{Cl} 1$. $15,1.51$ and $1.21 ; 95 \% \mathrm{Cl} 1.106,1.35$, interaction $P=0.32$ ) and whether done within $24 \mathrm{~h}$ or not within $24 \mathrm{~h}$ (respectively, HR 1.28; $95 \% \mathrm{Cl} 1.10,1.50$ and 1.23; $95 \% \mathrm{Cl} 1.10,1.38$, interaction $P=0.67$ ).
\end{abstract}

Conclusions: Among patients with ACS, lower in-hospital LVEF is associated with increased 1-year mortality or hospitalization for HF, regardless of the method or timing of the LVEF assessment. This has prognostic implications for clinical practice and suggests the possibility of using various methods of LVEF determination in clinical research.

Keywords: Acute coronary syndromes, Glucose-insulin-potassium, Left ventricular ejection fraction, Death, Hospitalization from heart failure

\section{Background}

Acute coronary syndrome (ACS) remains a leading cause of death in the United States [1]. Reduced left ventricular ejection fraction (LVEF), measured with standardized methods at 30 days following ACS, is an established marker for poor clinical outcome [2, 3], but the relationships between LVEF measured during

\footnotetext{
* Correspondence: hselker@tuftsmedicalcenter.org

${ }^{3}$ Center for Cardiovascular Health Services Research, Institute for Clinical Research and Health Policy Studies, Tufts Medical Center, 800 Washington St, \#63, Boston, MA 02111, USA

${ }^{5}$ Tufts Clinical and Translational Science Institute, Tufts University, Boston, MA, USA

Full list of author information is available at the end of the article
}

the index ACS hospitalization and clinical outcomes are less well-defined. Moreover, LVEF measured by noninvasive transthoracic echocardiography and invasive left ventriculography has been used to risk stratify patients with ACS [4-7], but the best modality and timing of measurement of in-hospital LVEF are not known.

In the IMMEDIATE (Immediate Myocardial Metabolic Enhancement During Initial Assessment and Treatment in Emergency care) Trial [8], participants with suspected ACS who received intravenous glucose-insulin-potassium 
(GIK) had generally fewer serious outcomes at 1 year than those treated with placebo, but the difference did not reach statistical significance [9]. However, among those presenting with ST elevation myocardial infarction (STEMI), the composite outcomes of cardiac arrest or 1-year mortality, and of cardiac arrest, mortality, or hospitalization for heart failure (HF), were significantly reduced [9]. Long-term outcomes based on LVEF routinely measured during ACS hospitalization, in general, and in the context of GIK, have not been studied. Using data from the IMMEDIATE Trial, we sought to determine if LVEF measured during ACS hospitalization was associated with the 1-year composite outcome of all-cause mortality or hospitalization for HF.

We hypothesized that reduced in-hospital LVEF would be a marker of increased risk of death or HF hospitalization at 1 year. As a secondary goal, we tested whether in-hospital LVEF was higher among IMMEDIATE Trial participants randomized to GIK as compared to those randomized to placebo.

\section{Methods}

\section{Study sample}

This study analyzed a subset of data on participants enrolled in the IMMEDIATE Trial $[8,10]$, a randomized, placebo-controlled, double-blind clinical effectiveness trial of GIK conducted from December 2006 through July 2011, in which paramedics, aided by electrocardiographbased decision support, enrolled 871 patients aged $\geq$ 30 years with high probabilities of ACS. Participants were given either GIK (30\% glucose, 50 U/L of regular insulin, and $80 \mathrm{mEq}$ of $\mathrm{KCl} / \mathrm{L}$ ) intravenously at $1.5 \mathrm{~mL} / \mathrm{kg} / \mathrm{h}$ for $12 \mathrm{~h}$, or identical-appearing placebo. This investigation included the subset of participants who had their LVEF measured during their index hospitalization by left ventriculography or echocardiography $[8,10]$.

Inclusion and exclusion criteria from the IMMEDIATE Trial Screened patients included all those transported by emergency medical services (EMS) in response to a 9-1-1 call for symptoms suggestive of ACS who were $\geq 30$ years of age and had an out-of-hospital electrocardiogram (ECG) performed. Inclusion was based on paramedics' clinical assessment of a patient likely having ACS, supplemented by decision support by the electrocardiographbased Acute Cardiac Ischemia Time-Insensitive Predictive Instrument (ACI-TIPI) and Thrombolytic Predictive Instrument (TPI). Patients were candidates for enrollment if the ACI-TIPI prediction of ACS was $75 \%$ or higher, if STEMI was detected by the TPI, and/or the patient met local standards for EMS identification of STEMI [10]. Patients were excluded if they had a language barrier or impaired reasoning, were prisoners or pregnant, or had clinically significant rales (Killip Class 3 or $4 \mathrm{HF})[8,10]$.

In addition, a National Institutes of Health appointed Data Safety Monitoring Board oversaw enrollment to ensure safe and ethical study conduct throughout the trial. Informed consent was obtained from all patients in accordance with the Exception from Informed Consent Requirements for Emergency Research per the Code of Federal Regulations 21 CFR 50.24 and included community consultation, institutional review board approval from all sites, assent from patients prior to randomization, and written consent once stabilized at the hospital [11].

The Coordinating Center's IRB at Tufts Medical Center provided approval for the overall trial along with all participating sites (University of Texas Southwestern Medical School, Dallas; Cambridge Health Alliance, Cambridge, Massachusetts; Alaska Regional Hospital, Anchorage; University of New Mexico School of Medicine, Albuquerque; Medical College of Wisconsin, Milwaukee; Medical Center of Central Georgia, Macon; Regions Hospital EMS, St. Paul, Minnesota; Avera Medical Group, Sioux Falls, South Dakota; Penn State Hershey Medical Center, Hershey, Pennsylvania; Emerson Hospital, Concord, Massachusetts; St Joseph Medical Center, Bellingham, Washington; Texas Tech University Health Sciences Center, El Paso; Yale New Haven Hospital, Connecticut)

\section{Data collection}

Data were collected by trained study staff. They were instructed to record LVEF as measured by cardiac catheterization; if the catheterization was not done, and LVEF was available by echocardiogram, this was recorded. The dates and times of echocardiograms were not routinely recorded, so medical records were reviewed to obtain them where possible. Based on emergency department (ED) presentation date and dates of LVEF measurement by left ventriculography or echocardiography, we classified a participant's LVEF as "early" (within $24 \mathrm{~h}$ of ED presentation), or "not early" if measured more than $24 \mathrm{~h}$ after ED presentation. We could not find the date and time of LVEF measurement for 94 out of 445 participants; for this study we assigned them to the not early group, as the majority (92) had echocardiograms, and our search showed that the majority of the echocardiograms were done more than $24 \mathrm{~h}$ after ED presentation.

\section{Data analysis}

Statistical analyses were performed using $R$, version 2.15.2. Tests were two-sided, using alpha $\leq 0.05$ as statistical significance. Comparison of means of in-hospital LVEF between the GIK and placebo groups, between early and not early LVEF measurement, and between catheterization and echocardiogram measurement all used two-sample student t-tests. Just for the Kaplan- 
Meier survival curves, they were plotted for participants with LVEFs in normal (55-70 \%), mildly abnormal (40-54 \%), moderately abnormal (25-39\%) and severely abnormal $(<25 \%)$ categories. Cox proportional hazards models were used to estimate univariate hazard ratios (HRs) for LVEF associated with the composite outcome of death or hospitalization for HF. Based on clinical and statistical significance in those analyses, we chose candidate variables for possible inclusion in a multivariable model to estimate adjusted HRs for the composite outcome of death or hospitalization for HF at 1 year. Using the final adjusted model, we also tested for dichotomous variables of timing of LVEF (early vs. not early) and the measurement method (catheterization vs. echocardiogram). Two-way interactions between LVEF and timing (early vs. not early) were tested in the Cox proportional hazard model of the composite outcome, adjusting for age and coronary artery disease (CAD). A similar analysis was done to test the two-way interaction between LVEF and test method (catheterization vs. echocardiogram). We checked assumptions needed for proportional hazards analyses by plotting and testing Schoenfeld residuals as related to time.

\section{Results}

\section{Baseline demographics}

Table 1 shows demographic and clinical features of participants having the composite outcome of death or hospitalization for HF at 1 year $(n=52)$ and those who did not $(n=393)$. Participants with the composite outcomes were older and more frequently had medical histories of CAD, HF, diabetes, hypertension, stroke, and hyperlipidemia. Rates of acute myocardial infarction were similar in both groups, but those participants that had the composite outcome presented with a significantly higher Killip Class. Please see Appendices below.

Data on LVEF measured by left ventriculography or echocardiography during the index hospitalization were available for 445 of 871 study participants. There were significant differences in baseline demographics and clinical characteristics of participants with and without in-hospital LVEFs recorded. Of participants with in-hospital LVEF measured, $92.6 \%$ had confirmed diagnoses of ACS, compared with $33.1 \%$ among those without measured LVEF. There were no significant differences between characteristics of participants who had in-hospital LVEF recorded by left ventriculography vs. echocardiography. For 248 participants, LVEF was measured early (219 [80.1\%] by catheterization), defined as measurement within $24 \mathrm{~h}$ of presentation, and for 197 participants, the LVEF measurement was performed not early (54 [19.7 \%] by left ventriculography).

\section{Univariate models of LVEF and outcomes}

Participants with the lowest LVEF had the lowest survival and the greatest incidence of composite outcomes (Fig. 1). Univariate Cox proportional hazard models were used to identify factors associated with the composite outcome (Table 2). A reduction of LVEF by $5 \%$ decrements was significantly associated with higher hazards of death or hospitalization for $\mathrm{HF}$ over 1 year $(\mathrm{HR}=1.31,95 \% \mathrm{CI}$ : 1.20-1.45, $P<0.001)$. Also significant was age, expressed in 10-year increments (HR $=1.79,95 \%$ CI: $1.44-2.24, P<$ $0.001)$, prior $\mathrm{CAD}(\mathrm{HR}=4.46,95 \% \mathrm{CI}: 2.47-8.04, P<$ $0.001)$, HF (HR $=3.56,95 \% \mathrm{CI}: 1.86-6.79, P<0.001)$, diabetes (HR $=1.88,95 \% \mathrm{CI}: 1.06-3.34, P=0.030)$, hypertension $(\mathrm{HR}=2.17,95 \% \mathrm{CI}: 1.12-4.23, P=0.022)$, and stroke $(\mathrm{HR}=2.56,95 \% \mathrm{CI}: 1.20-5.43, P=0.015)$.

\section{Multivariable models of LVEF and outcomes}

Multivariate Cox models were used to better characterize the association of LVEF with the composite outcome. In stepwise regression, candidate parameters for inclusion included LVEF expressed by $5 \%$ decrements; age, sex, history of CAD, HF and diabetes. The model that had the lowest akaike information criteria (best fit) following the selection process included only LVEF, age, and CAD. The $\mathrm{C}$ statistic (which is equivalent to the receiver-operating characteristic (ROC) curve area) for that model is 0.805 $(\mathrm{SE}=0.04)$.

The effect of $5 \%$ lower LVEF on 1-year death or hospitalization for HF remained statistically significant after adjusting for age and history of CAD $(\mathrm{HR}=1.26$; $95 \%$ CI: $1.15-1.38, P<0.001)$ when LVEF was used as a continuous variable. When LVEF was tested as a binary variable and dichotomized, participants with LVEF $<40 \%$ had higher hazard of 1-year mortality and hospitalization for HF compared to participants with LVEF $\geq 40 \%$, after adjusting for age and history of CAD ( $\mathrm{HR}=3.59 ; 95 \% \mathrm{CI}$ : 2.05-6.27, $P<0.001)$. The proportional hazard assumption was not violated. Separating the composite outcome into its components, reductions of LVEF by $5 \%$ decrements were also significantly associated with higher hazard of death and hospitalization for HF when adjusted for age and history of CAD (HR $=1.18$; $95 \%$ CI: $1.06-1.32, P=$ 0.002 ; and $\mathrm{HR}=1.37$; $95 \% \mathrm{CI}: 1.18-1.58, P<0.001$, respectively) (Table 2).

The HRs for the association of LVEF with the study outcomes were similar whether measured by left ventriculography or by echocardiography, (respectively, HR = 1.32; $95 \%$ CI 1.15, 1.51 and 1.21; $95 \%$ CI 1.106, 1.35) and whether done within $24 \mathrm{~h}$ or not within $24 \mathrm{~h}$ (respectively, $\mathrm{HR}=1.28 ; 95 \% \mathrm{CI} 1.10,1.50$ and 1.23; $95 \%$ CI 1.10, 1.38). Tests for interactions of LVEF and measurement method and LVEF and timing did not reach significance (Table 3). Controlling for method of LVEF measurement (left ventriculography vs. echocardiography) 
Table 1 Baseline characteristics of participants with LVEF and outcomes ( $N=445)$

\begin{tabular}{|c|c|c|}
\hline Variables & $\begin{array}{l}\text { Participants with neither death nor hospitalization } \\
\text { for HF at } 1 \text { year } N=393\end{array}$ & $\begin{array}{l}\text { Participants with death or hospitalization } \\
\text { for } \mathrm{HF} \text { at } 1 \text { year } N=52\end{array}$ \\
\hline Age (mean $\pm S D$, years) & $61 \pm 12.1(393)$ & $71 \pm 13.0(52)$ \\
\hline Gender, \% Male & $75.6(297)$ & $69.2(36)$ \\
\hline White race (vs. non- White race) & $86.7 \%(341)$ & $88.5 \%(42)$ \\
\hline Body Mass Index, (mean \pm SD, units) & $28.9 \pm 6.3(372)$ & $27.9 \pm 6.0(42)$ \\
\hline \multicolumn{3}{|c|}{ Time from onset of symptoms to treatment (minutes) } \\
\hline Mean $\pm S D$ & $146.8 \pm 206.4(353)$ & $111.7 \pm 109.3(37)$ \\
\hline median $<\mid \mathrm{QR}>$ & $78<50.0-140.0>$ & $65<40.0-145.0>$ \\
\hline \multicolumn{3}{|l|}{ Chief Complaint on Presentation } \\
\hline Chest Pain & $91.8 \%(361)$ & $84.6 \%(44)$ \\
\hline \multicolumn{3}{|l|}{ Medical History, \% (n) } \\
\hline CAD (Ml, $\mathrm{PCl}$ or $\mathrm{CABG})$ & $30.8 \%(121)$ & $69.2 \%(36)$ \\
\hline Heart Failure & $6.7 \%(25)$ & $23.1 \%(12)$ \\
\hline Diabetes Mellitus & $21.1 \%(83)$ & $34.7 \%(18)$ \\
\hline Hypertension & $62.3 \%(245)$ & $78.8 \%(41)$ \\
\hline Stroke & $5.9 \%(23)$ & $15.4 \%(8)$ \\
\hline Hyperlipidemia & $46.8 \%(184)$ & $65.4 \%(34)$ \\
\hline \multicolumn{3}{|l|}{ Hospital Reperfusion Treatment, \%(n) } \\
\hline $\mathrm{PCl}$ & $73.8 \%(290)$ & $59.6 \%(31)$ \\
\hline Thrombolytic therapy & $0.8 \%(3)$ & $9.6 \%(5)$ \\
\hline CABG & $5.1 \%(20)$ & $7.7 \%(4)$ \\
\hline \multicolumn{3}{|l|}{ Confirmed Diagnosis, \% (n) } \\
\hline Acute Myocardial Infarction & $80.4 \%$ (316) & $78.8 \%(41)$ \\
\hline \multicolumn{3}{|l|}{ Killip Class } \\
\hline 1 & $76.1 \%(299)$ & $51.9 \%(27)$ \\
\hline 2 & $1.8 \%(7)$ & $1.3 \%(7)$ \\
\hline 3 & $0.5 \%(2)$ & $3.8 \%(2)$ \\
\hline 4 & $2.0 \%(8)$ & $9.6 \%(5)$ \\
\hline Unstable Angina & $12.7 \%(50)$ & $9.6 \%(5)$ \\
\hline \multicolumn{3}{|l|}{ ECG based findings } \\
\hline ST Elevation on Presentation (ECG) & $70.8 \%(264)$ & $66.0 \%(33)$ \\
\hline ACI-TIPI score, (Mean \pm SD) & $80.7 \pm 17.6(386)$ & $84.8 \pm 11.2(49)$ \\
\hline LVEF in \%, Median (IQR) & $50(40,60)$ & $35(25,45)$ \\
\hline GIK (vs. Placebo) & $47.6 \%$ (187) & $38.5 \%$ (20) \\
\hline
\end{tabular}

or timing of measurement (early vs. not early) did not alter the association of LVEF with the outcome (Table 4). Additionally, while having an echocardiogram (vs. left ventriculography) and being not early (vs. early) were associated with the 1-year outcome, neither was statistically significant.

Sensitivity analyses were performed in which we reestimated HRs for LVEF, adjusting not only for already specified covariates age and CAD history, but also for sex, HF history, and diabetes. Reductions of LVEF by $5 \%$ were significantly associated with higher hazard of the 1-year composite outcome $(\mathrm{HR}=1.25 ; 95 \% \mathrm{CI}$ : 1.14-1.38, $P<0.001)$. Also, the HRs for the association of LVEF with the study outcome when measured by left ventriculography vs. by echocardiography, and for when measured early vs. not early, were similar. Finally, we studied the association of the IMMEDIATE Trial treatment, GIK vs. placebo, on in-hospital LVEF, stratified by method (catheterization vs. echocardiogram) and timing (early vs. not early. There was a trend toward higher inhospital LVEF among those receiving GIK. When measured by echocardiography alone, median LVEF with 


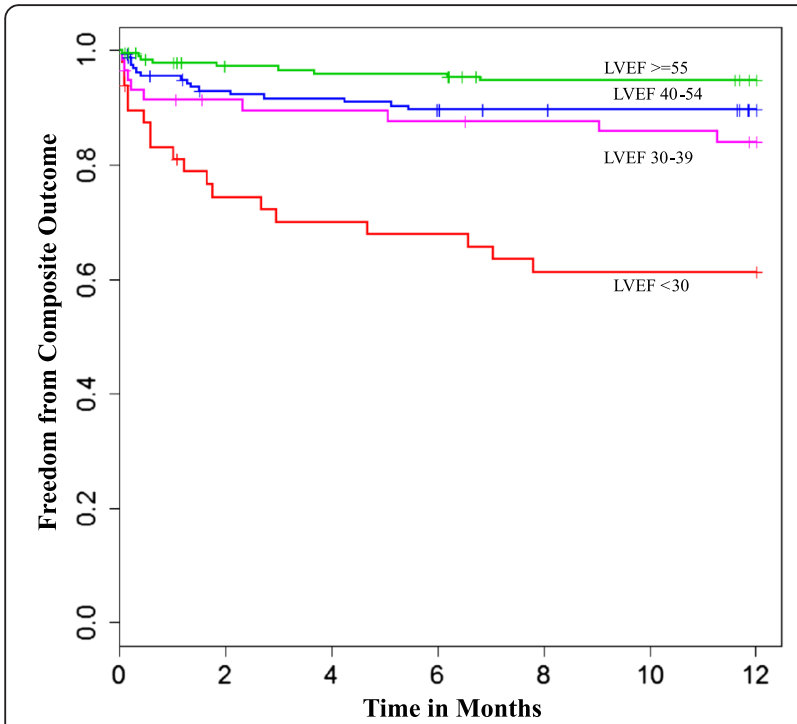

Fig. 1 Kaplan-Meier plot for categories of LVEF

GIK $50 \%$ vs $45 \%$ with placebo $(P=0.08)$. When measured by either echocardiography or catheterization, the median LVEF with GIK was $50 \%$ vs $45 \%$ with placebo (P-0.07) (Table 5).

\section{Discussion}

In this post hoc analysis of IMMEDIATE trial participants who had in-hospital LVEF assessment we demonstrate that in-hospital LVEF for patients with ACS can identify patients at risk for death or HF hospitalization within 1 year. Our results do not favor left ventriculography or echocardiography as a preferred modality for LVEF measurement; as the predictive ability of LVEF was similar for both modalities. These results also do not support a need for LVEF assessment within $24 \mathrm{~h}$ of presentation. These results have practical importance for the care of patients with ACS because our data suggest that assessment of LVEF measured at any point in the hospitalization can be used to predict patients at risk of death or hospitalization for HF within 1 year.

In-hospital LVEF assessment is not routinely performed in ACS patients. One study demonstrated over $40.8 \%$ of patients with non-ST elevation myocardial infarction did not have an LVEF assessment [12,13], and the percentage of patients getting LVEF measurement has steadily increased in the last two decades $[14,15]$. LVEF is superior to end systolic volume index and infarct size in predicting 6-month mortality after myocardial infarction [2]. The presence of LV dysfunction on baseline left ventriculography in patients enrolled in the HORIZONS-AMI trial who underwent primary PCI was a powerful predictor of early and late mortality irrespective of the extent of coronary artery disease [7]. The age, creatinine and ejection fraction score (ACEF), as proposed by J H Lee et al. may
Table 2 Unadjusted and multivariable-adjusted Cox proportionate hazard models for composite outcomes (death and hospitalization for HF) by LVEF measured in-hospital ( $N=445$, no of events $=52)$

Unadjusted cox proportional hazard model

Variables

HR $(95 \% \mathrm{Cl}) \quad P$-value

LVEF/5 \% decrease

$1.31(1.20,1.45) \quad<0.001$

Age/10 years increase

$1.79(1.44,2.24)<0.001$

Gender, Male

$1.37(0.41,1.32) \quad 0.23$

White race (nonwhite race reference)

$1.13(0.48,2.65) \quad 0.77$

Body Mass Index

$0.97(0.92,1.02) \quad 0.32$

Time from onset of symptoms to treatment* $0.98(0.99,1.00) \quad 0.33$

Chief complaint on presentation

$\begin{array}{lll}\text { Chest pain } & 0.53(0.23,1.12) & 0.10\end{array}$

Out of Hospital ECG ST-Elevation $\quad 0.84(0.47,1.50) \quad 0.55$

ACI-TIPI Score

$1.02(0.99,1.04) \quad 0.12$

Medical history

CAD (MI, PCl or CABG)

Heart Failure

$4.46(2.47,8.04)<0.001$

Diabetes

$3.56(1.86,6.79)<0.001$

Hypertension

$1.88(1.06,3.34) \quad 0.03$

Stroke

$2.17(1.12,4.23) \quad 0.02$

$2.56(1.20,5.43) \quad 0.02$

Multivariable adjusted cox proportional hazard models

Variables

HR $(95 \% \mathrm{Cl}) \quad P$ value

Outcome (Composite)

LVEF/5 \% lower (combined)

$1.26(1.15,1.38)<0.001$

Age/10 years Increase

$1.73(1.38,2.18)<0.001$

History of $\mathrm{CAD}(\mathrm{Ml}, \mathrm{PCl}$, or $\mathrm{CABG})$

$2.97(1.62,5.43)<0.001$

Outcome (Death)

LVEF/5 \% lower (combined)

$1.18(1.06,1.32) \quad 0.002$

Age/10 years Increase

$1.94(1.47,2.55) \quad<0.001$

History of $\mathrm{CAD}(\mathrm{Ml}, \mathrm{PCl}$, or $\mathrm{CABG})$

$3.56(1.69,7.49)<0.001$

Outcome (Hospitalization for HF)

LVEF/5 \% lower (combined)

$1.37(1.18,1.58)<0.001$

Age/10 years Increase

$1.35(0.95,1.91) \quad 0.09$

History of $\mathrm{CAD}(\mathrm{Ml}, \mathrm{PCl}$, or $\mathrm{CABG})$

$2.27(0.93,5.56) \quad 0.007$

Table 3 Association of LVEF with composite outcome, stratified by timing and type of procedure

\begin{tabular}{lll}
\hline Variable & $\begin{array}{l}\text { Adjusted HR } \\
(95 \% \mathrm{Cl})\end{array}$ & $\begin{array}{l}\text { Interaction } \\
P \text { value }\end{array}$ \\
\hline LVEF- by catheterization/5 \% lower $^{\mathrm{a}}$ & $1.32(1.15,1.51)$ & 0.32 \\
LVEF- by echocardiogram/5 \% lower $^{\mathrm{a}}$ & $1.20(1.06,1.35)$ & \\
LVEF- early/5 \% lower $^{\mathrm{a}}$ & $1.28(1.10,1.50)$ & 0.67 \\
LVEF- not early/5 \% lower* $^{*}$ & $1.23(1.10,1.38)$ & \\
\hline
\end{tabular}

${ }^{a}$ Adjusted for age and history of CAD 
Table 4 Model for LVEF measured by cardiac catheterization (reference echocardiogram) and not early (reference Early) $(N=445$, no of events $=52)$

\begin{tabular}{lll}
\hline Variable & $\begin{array}{l}\text { HR } \\
(95 \% \mathrm{Cl})\end{array}$ & $P$ value \\
\hline LVEF/5 \% lower (combined) & 1.25 & $<0.001$ \\
& $(1.14,1.37)$ & \\
Age/10 years Increase & 1.71 & $<0.001$ \\
& $(1.36,2.15)$ & \\
History of CAD (MI, PCl, or CABG) & 2.94 & $<0.001$ \\
& $(1.60,5.40)$ & \\
LVEF - echocardiogram & 1.62 & 0.08 \\
(reference catheterization) & $(0.93,2.80)$ & \\
LVEF/5 \% lower (combined) & 1.25 & $<0.001$ \\
& $(1.13,1.36)$ & \\
Age/10 years Increase & 1.71 & $<0.001$ \\
History of CAD (MI, PCl, or CABG) & $(1.36,2.15)$ & \\
& 2.86 & $<0.001$ \\
LVEF - not early (reference early) & $(1.56,5.26)$ & \\
& 1.53 & 0.14 \\
\hline
\end{tabular}

be used to stratify the 1-year mortality risk in 30-day survivors who underwent percutaneous coronary intervention (PCI) after acute myocardial infarction [16]. In spite of optimal medical therapy and clinically driven percutaneous coronary intervention (PCI), LVEF and angiographic burden of disease at baseline retain prognostic importance for patients aggressively treated for stable CAD [17].

Our study, which featured the full spectrum of ACS presentations, suggests that useful information can be obtained by measuring LVEF by either left ventriculography or echocardiography at any time prior to hospital discharge. These findings have important implications for clinical effectiveness research, because we demonstrate the utility of collecting data from clinically-indicated and

Table 5 Distribution of LVEF \% by experimental treatment groups

\begin{tabular}{lcll}
\hline Variable & GIK, & Placebo & $P$ value \\
& Mean (SD) & Mean (SD) & for Mean \\
& Median & Median & \\
& LVEF\%, (N) & LVEF\%, (N) & \\
\hline LVEF (Catheterization and & $48.6(14)$ & $46.2(14)$ & 0.07 \\
Echocardiogram) & $50(207)$ & $45(238)$ & \\
LVEF (Catheterization) & $49.3(14)$ & $48.3(14)$ & 0.33 \\
& $50(120)$ & $50(153)$ & \\
LVEF (Echocardiogram) & $47.6(14)$ & $43.6(14)$ & 0.08 \\
& $50(87)$ & $45(85)$ & \\
LVEF (Early ${ }^{a}$ ) & $49.2(13)$ & $47.4(13)$ & 0.27 \\
& $50(110)$ & $50(138)$ & \\
LVEF (Not early ${ }^{\text {b }}$ & $47.8(15)$ & $44.6(15)$ & 0.13 \\
& $50(97)$ & $45(100)$ & \\
\hline
\end{tabular}

${ }^{a}$ Early when the LVEF was measured within 0 days of ED arrival ${ }^{b}$ Not early when LVEF was measured 1 or more days after ED arrival or with unknown dates performed LVEF assessments in large multicenter clinical trials as a means to define the risk of longitudinal cardiac outcomes. If substantiated as a research tool, this finding has the potential to reduce the need for core lab LVEF assessments, which can be quite costly.

In the IMMEDIATE Trial, GIK did not significantly reduce the primary endpoint of progression of unstable angina to AMI, but it did significantly reduce the composite endpoint of cardiac arrest or mortality, and in the biological mechanism cohort, the median infarct size measured by sestamibi SPECT imaging at 30 days was 80 \% lower in the GIK compared to the placebo group. Further, there was a trend for better LVEF measured by SPECT imaging at 30 days with GIK [8]. Our study also showed that GIK had a trend toward improved in-hospital LVEF when compared to placebo especially when measured by echocardiography. Future well-powered studies will be required to further delineate the effect of GIK on infarct size and long-term left ventricular function.

Our study has several limitations. It is a retrospective analysis using data from a prospective randomized controlled trial on a subset with 445 participants who had LVEF measured by echocardiogram or cardiac catheterization. While the sample is not large, we had 52 composite outcomes in the group. The study cohort includes patients with high-risk ACS. The LVEF was measured by two different modalities at slightly different times, which could introduce bias since the LVEF measured by left ventriculography was during revascularization and LVEF measured by echocardiography was primarily obtained prior to hospital discharge. However, our study showed that the methodology and the timing of the LVEF measurement did not matter. The study cannot compare the in-hospital LVEF measurement with an assessment made by a core lab, and hence we cannot say that clinical LVEF results necessarily give the same information as measurements made by a core laboratory. However, we did find that LVEF measured in the hospital and not by a core laboratory was significantly associated with 1-year outcomes of death and HF hospitalization.

\section{Conclusion}

Lower in-hospital LVEF is associated with higher rates of 1-year mortality and hospitalization from HF in patients hospitalized with ACS, regardless of the method or timing of LVEF assessment. This has prognostic implications for clinical practice and suggests the possibility of using various methods of LVEF determination in clinical research.

\section{Abbreviations}

ACI-TIPI, Acute Cardiac Ischemia Time-Insensitive Predictive Instrument; $A C S$, acute coronary syndrome; CAD, coronary artery disease; ED, emergency department; GIK, glucose-insulin-potassium; HR, hazard ratio; HF, heart failure; 
LVEF, left ventricular ejection fraction; STEMI, ST elevation myocardial infarction; TPI, Thrombolytic Predictive Instrument.

\section{Appendices}

\section{A. Baseline characteristics}

Figure 1. Participants with LVEF and outcomes

Table 1. Baseline characteristics of participants with and without LVEF

Table 2. Baseline characteristics of participants with LVEF measured by catheterization or echocardiogram Table 3. Baseline characteristics of participants with LVEF measured early or not early

Table 4. Difference in days by catheterization and echocardiogram

\section{B. Intermediate models}

Table 1. Composite outcome and LVEF measured regardless of modality and timing

Table 2. Composite outcome and LVEF measured by catheterization and echocardiogram

Table 3. Composite outcome and LVEF measured early and not early

\section{Sensitivity analyses}

Figure 1. Kaplan-Meir survival curve for LVEF $<40 \%$ \& $\geq 40 \%$

Table 1. Composite outcome in participants with LVEF $<40 \% \& \geq 40 \%$

Table 2. Composite outcome and LVEF measured in participants who had PCI

Table 3. Composite outcome and LVEF measured in participants who had confirmed MI

\section{Acknowledgements}

Angie Mae Rodday: statistical analysis. Nina Bonnoyer and Cathy Griffin: administrative and technical support.

\section{Funding}

The IMMEDIATE Trial was funded by the National Institutes of Health cooperative agreement from National Heart, Lung and Blood Institute (U01HL077821, U01HL077823, and U01HL077826). The IMMEDIATE Trial is registered at www.ClinicalTrials.gov (NCT00091507).

This study was funded by a NRSA/AHRQ T32 training grant (5T32HS000060-20).

\section{Availability of data and materials}

All clinical datasets are available for review in accordance with the study sponsor, the National Institutes of Health.

\section{Author's contributions}

JTM: conception and design, analysis and interpretation of data, statistical analysis, drafting, revising and finalizing the manuscript. JRB: obtaining funding, conception and design, drafting, revising, critical revision of manuscript for important intellectual content, administrative support. RR: conception and design, statistical analysis, drafting, revising and finalizing the manuscript. HF: obtaining funding, design, statistical analysis, critical revision of manuscript for important intellectual content. MR: design, statistical analysis, critical revision of manuscript for important intellectual content. DK: design, critical revision of manuscript for important intellectual content, supervision. WJM: design, critical revision of manuscript for important intellectual content, supervision. GJH: conception and design, analysis and interpretation of data, drafting, revising and finalizing the manuscript, supervision. HPS: conception and design, analysis and interpretation of data, drafting, revising and finalizing the manuscript, critical revision of manuscript for important intellectual content, administrative support, overall supervision. All authors read and approved the final manuscript.

\section{Competing interests}

The authors declare that they have no competing interests.

\section{Author details}

${ }^{1}$ Clinical and Translational Science Graduate Program, Sackler School of Biomedical Sciences, Tufts Clinical and Translational Science Institute, Tufts University, Boston, MA, USA. ${ }^{2}$ MCRI Center for Translational Genomics, Molecular Cardiology Research Institute, Tufts Medical Center, Boston, MA, USA. ${ }^{3}$ Center for Cardiovascular Health Services Research, Institute for Clinical Research and Health Policy Studies, Tufts Medical Center, 800 Washington St, \#63, Boston, MA 02111, USA. ${ }^{4}$ Department of Medicine (Cardiovascular Division) and Radiology, Beth Israel Deaconess Medical Center Harvard Medical School, Boston, MA, USA. ${ }^{5}$ Tufts Clinical and Translational Science Institute, Tufts University, Boston, MA, USA. ${ }^{6}$ College of Pharmacy, King Saud University, Riyadh, Saudi Arabia. ${ }^{7}$ Regis College, Weston, MA, USA. ${ }^{8}$ Riverside Methodist Hospital, Ohio Health Heart and Vascular Physicians, Columbus, $\mathrm{OH}, \mathrm{USA}$.

Received: 26 January 2016 Accepted: 22 June 2016

Published online: 03 August 2016

References

1. American Heart Association. Heart and stroke statistics. Circulation. 2013; 127(1):e6-245.

2. Burns RJ, Gibbons RJ, Yi Q, et al. The relationships of left ventricular ejection fraction, end-systolic volume index and infarct size to six-month mortality after hospital discharge following myocardial infarction treated by thrombolysis. J Am Coll Cardiol. 2002:39:30-6.

3. Zaret BL, Wackers FJ, Terrin ML, et al. Value of radionuclide rest and exercise left ventricular ejection fraction in assessing survival of patients after thrombolytic therapy for acute myocardial infarction: Results of thrombolysis in myocardial infarction (timi) phase ii study. The timi study group. J Am Coll Cardiol. 1995;26:73-9.

4. Nicolosi GL, Latini R, Marino P, et al. The prognostic value of predischarge quantitative two-dimensional echocardiographic measurements and the effects of early lisinopril treatment on left ventricular structure and function after acute myocardial infarction in the gissi-3 trial. Eur Heart J. 1996;17:1646-56.

5. Nishimura RA, Reeder GS, Miller F, et al. Prognostic value of predischarge two-dimensional echocardiogram after acute myocardial-infarction. Am J Cardiol. 1984:53:429-32.

6. Penco M, Sciomer S, Vizza CD, et al. Clinical impact of echocardiography in prognostic stratification after acute myocardial infarction. Am J Cardiol. 1998:81:17G-20.

7. Daneault B, Genereux P, Kirtane AJ, et al. Comparison of three-year outcomes after primary percutaneous coronary intervention in patients with left ventricular ejection fraction $<40 \%$ versus $>/=40 \%$ (from the horizons-ami trial). Am J Cardiol. 2013;111:12-20.

8. Selker HP, Beshansky JR, Sheehan PR, et al. Out-of-hospital administration of intravenous glucose-insulin-potassium in patients with suspected acute coronary syndromes: The immediate randomized controlled trial. JAMA t. 2012:307:1925-33.

9. Selker HP, Udelson JE, Massaro JM, et al. One-year outcomes of out-ofhospital administration of intravenous glucose, insulin, and potassium (gik) in patients with suspected acute coronary syndromes (from the immediate trial). Am J Cardiol. 2014;113:1593-776.

10. Selker HP, Beshansky JR, Griffith JL, et al. Study design for the immediate myocardial metabolic enhancement during initial assessment and treatment in emergency care (immediate) trial: a double-blind randomized controlled trial of intravenous glucose, insulin, and potassium for acute coronary syndromes in emergency medical services. Am Heart J. 2012;163:315-22.

11. Beshansky JR, Sheehan PR, Klima KJ, et al. A community consultation survey to evaluate support for and success of the IMMEDIATE trial. Clinical Trials. 2014;11:178-86 
12. Jedrzkiewicz S, Goodman SG, Yan RT, et al. Evaluation of left ventricular ejection fraction in non-st-segment elevation acute coronary syndromes and its relationship to treatment. Am Heart J. 2010;159:605-11.

13. Hernandez AF, Velazquez EJ, Solomon SD, et al. Left ventricular assessment in myocardial infarction - the valiant registry. Arch Intern Med. 2005;165:2162-9.

14. Santolucito PA, Tighe DA, Lessard D, et al. Changing trends in the evaluation of ejection fraction in patients hospitalized with acute myocardial infarction: The worcester heart attack study. Am Heart J. 2008;155:485-93.

15. Lopez-Jimenez F, Goraya TY, Hellermann JP, et al. Measurement of ejection fraction after myocardial infarction in the popoulation. Chest. 2004;125:397-403.

16. Lee JH, Bae MH, Yang DH, et al. Prognostic value of the age, creatinine, and ejection fraction score for 1-year mortality in 30-day survivors who underwent percutaneous coronary intervention after acute myocardial infarction. Am J Cardiol. 2015;1 15(9):1167-73.

17. Mancini GB, Hartigan PM, Bates ER, et al. Prognostic importance of coronary anatomy and left ventricular ejection fraction despite optimal therapy: assessment of residual risk in the clinical outcomes utilizing revascularization and aggressive drug evaluation trial. Am Heart J. 2013:166:481-7.

\section{Submit your next manuscript to BioMed Central} and we will help you at every step:

- We accept pre-submission inquiries

- Our selector tool helps you to find the most relevant journal

- We provide round the clock customer support

- Convenient online submission

- Thorough peer review

- Inclusion in PubMed and all major indexing services

- Maximum visibility for your research

Submit your manuscript at www.biomedcentral.com/submit 